\title{
Peripheral nerve grafts implanted into the substantia nigra in patients with Parkinson's disease during deep brain stimulation surgery: 1-year follow-up study of safety, feasibility, and clinical outcome
}

\author{
Craig G. van Horne, MD, PhD,1-3 Jorge E. Quintero, PhD, 1,3 John T. Slevin, MD, MBA, 1,4 \\ Amelia Anderson-Mooney, PhD, ${ }^{1,2,4}$ Julie A. Gurwell, PA-C, PhD, ${ }^{1,4}$ Andrew S. Welleford, BS, ${ }^{1,3}$ \\ John R. Lamm, MD, ${ }^{1,2}$ Renee P. Wagner, RN, ${ }^{4}$ and Greg A. Gerhardt, PhD ${ }^{1-4}$ \\ ${ }^{1}$ Brain Restoration Center and Departments of ${ }^{2}$ Neurosurgery, ${ }^{3}$ Neuroscience, and ${ }^{4}$ Neurology, University of Kentucky, Lexington, \\ Kentucky
}

\begin{abstract}
OBJECTIVE Currently, there is no treatment that slows or halts the progression of Parkinson's disease. Delivery of various neurotrophic factors to restore dopaminergic function has become a focus of study in an effort to fill this unmet need for patients with Parkinson's disease. Schwann cells provide a readily available source of such factors. This study presents a 12-month evaluation of safety and feasibility, as well as the clinical response, of implanting autologous peripheral nerve grafts into the substantia nigra of patients with Parkinson's disease at the time of deep brain stimulation (DBS) surgery.
\end{abstract}

METHODS Standard DBS surgery targeting the subthalamic nucleus was performed in 8 study participants. After DBS lead implantation, a section of the sural nerve containing Schwann cells was harvested and unilaterally grafted to the substantia nigra. Adverse events were continually monitored. Baseline clinical data were obtained during standard preoperative evaluations. Clinical outcome data were obtained with postoperative clinical evaluations, neuropsychological testing, and MRI at 1 year after surgery.

RESULTS All 8 participants were implanted with DBS systems and grafts. Adverse event profiles were comparable to those of standard DBS surgery with the exception of 1 superficial infection at the sural nerve harvest site. Three participants also reported numbness in the distribution of the sural nerve distal to the harvest site. Motor scores on Unified Parkinson's Disease Rating Scale (UPDRS) part III while the participant was off therapy at 12 months improved from baseline (mean \pm SD $25.1 \pm 15.9$ points at 12 months vs $32.5 \pm 9.7$ points at baseline). An analysis of the lateralized UPDRS scores also showed a greater overall reduction in scores on the side contralateral to the graft.

CONCLUSIONS Peripheral nerve graft delivery to the substantia nigra at the time of DBS surgery is feasible and safe based on the results of this initial pilot study. Clinical outcome data from this phase I trial suggests that grafting may have some clinical benefit and certainly warrants further study to determine if this is an efficacious and neurorestorative therapy.

Clinical trial registration no.: NCT01833364 (clinicaltrials.gov)

https://thejns.org/doi/abs/10.3171/2017.8.JNS163222

KEY WORDS deep brain stimulation; cell therapy; multimodal DBS; neurotrophic factor; functional neurosurgery

$\mathrm{T}$ HE motor symptoms of Parkinson's disease (PD) are primarily related to the degeneration of dopaminergic neurons within the midbrain. One of the hallmarks of the disease is the continual progression of symptoms associated with ongoing cell loss. Although medical and surgical therapies can ameliorate some of these symptoms, disease progression leads to treatmentresistant symptoms that are often accompanied by troublesome fluctuations and medication side effects. Unfortunately, no treatment slows or halts disease progression.

ABBREVIATIONS CID = clinically important difference; DBS = deep brain stimulation; DSMB = Data Safety Monitoring Board; GDNF = glial cell-derived neurotrophic factor; LED = levodopa equivalent dose; PD = Parkinson's disease; ROI = region of interest; STN = subthalamic nucleus; SWI = susceptibility-weighted imaging; UPDRS = Unified Parkinson's Disease Rating Scale.

SUBMITTED January 3, 2017. ACCEPTED August 8, 2017.

INCLUDE WHEN CITING Published online February 16, 2018; DOI: 10.3171/2017.8.JNS163222. 
Thus, altering the course of clinical decline in PD is perhaps the largest unmet need facing patients, researchers, and clinicians. ${ }^{17}$

The majority of surgical research efforts to alter disease progression have focused on either cell replacement strategies or the delivery of growth factors that target the restoration of dopaminergic neurons. Transplantation of fetal dopaminergic tissue has not demonstrated significant clinical improvement in randomized double-blinded control studies. ${ }^{28}$ However, pronounced long-term improvements in several patients ${ }^{2}$ have prompted a new round of investigations in Europe (TRANSEURO; clinicaltrials. gov database registration no.: NCT01898390), despite ongoing moral and ethical issues surrounding the use of human fetal tissue as donor material. Meanwhile, strategies for growth factor delivery have focused on the use of a single factor and have included direct infusion of the factor, such as glial cell-derived neurotrophic factor (GDNF), ${ }_{12,35}$ or the infusion of a viral vector, such as AAV-neurturin, capable of initiating the production of the factor. ${ }^{3}$ Intraparenchymal GDNF delivery has shown promise in preclinical studies that failed to show significance in a phase II trial. ${ }^{21}$ Despite promising early results, 2 randomized controlled studies with AAV-2 neurturin (CERE-120) did not meet their primary end points..$^{24,25}$

These clinical studies have clearly identified some of the hurdles in developing neurorestorative therapies. Identifying the right factor(s), target(s), and methodology for optimum delivery poses major challenges. An additional consideration is that patients who are appropriate for enrollment in surgical implantation therapy trials are often at a stage in their disease where deep brain stimulation (DBS) is now the standard of care. This is especially relevant for studies aimed at changing disease progression. From a patient-centric view, choosing to participate in a long-term trial to assess alteration of disease progression may mean foregoing the benefits of DBS. For a study that may last 1,2 , or more years, having patients with PD forego the standard of care for so long for unproven therapies creates a set of ethical dilemmas.

Considering these challenges, we have identified a new approach to investigate the ability to alter disease progression. We use a cell-based graft that consists of peripheral nerve tissue as a source of growth factors and growth-promoting proteins for delivery into the parenchymal region with dopaminergic cell loss. We deploy the tissue grafts in the substantia nigra immediately following stimulating electrode placement into the subthalamic nucleus (STN) in patients with PD undergoing DBS surgery.

Peripheral nerve tissue is an abundant source of Schwann cells, which support and maintain axonal function and metabolism. ${ }^{18}$ Following injury to the nerve, Schwann cells transdifferentiate into repair cells and support axonal regeneration in the peripheral nervous system. ${ }^{16}$ The specific mechanism involves the activation of the transcription factor, c-Jun, which enables transdifferentiation through the upregulation of the production and release of growth factors, ${ }^{36}$ the downregulation of regulatory maintenance programs, the reentry of the cell into the cell cycle, and the initiation of chemical signaling in order to attract the migration of macrophages to assist in the pro- cess of regeneration. ${ }^{1}$ The growth factors produced include those that have been shown to promote and maintain dopaminergic neurons in experimental conditions: GDNF, ${ }^{15}$ brain-derived neurotrophic factor, ${ }^{26}$ and neurotrophin $3 .{ }^{11}$

We previously reported the safety and feasibility of grafting autologous peripheral nerve tissue into the substantia nigra. ${ }^{38}$ This study presents the full experience of the clinical trial that enrolled 8 participants and the clinical outcome data at 1-year postimplantation.

\section{Methods}

This study was designed as a 12-month, investigatorinitiated, open-label, single-center, phase I trial to assess safety and feasibility as the primary end points. This study was registered with (registration no. NCT01833364, clinicaltrials.gov). Clinical data were collected to evaluate the potential of the graft to affect the clinical course of PD symptoms. This study was approved by the University of Kentucky institutional review board, and informed consent was obtained from all study participants.

\section{Patient Selection}

Eight patients with a diagnosis of idiopathic PD, who had been selected and provided consent to receive bilateral DBS of the STN, were informed about the study and gave both assent and formal consent to participate. Inclusion criteria were age 40-75 years; greater than 5-year history of symptomatic, idiopathic PD; and an ability to participate in all follow-up appointments. As part of our standard preoperative DBS protocol, participants were evaluated using the Unified Parkinson's Disease Rating Scale (UPDRS), both on and off medication, and underwent a formal neuropsychological evaluation. The Parkinson's Disease Questionnaire-8 was used to assess overall quality of life.

\section{Safety and Feasibility}

Patients were monitored for both perioperative and long-term postoperative adverse events. Clinical monitoring included the perioperative time points through the conclusion of the study at the 12-month time point. During this time period, adverse events were recorded from clinical reports, chart reviews, and specific queries during the clinical visits. All adverse events were submitted to and reviewed by the Data and Safety Monitoring Board (DSMB) at the University of Kentucky. Adverse events were graded as mild, moderate, or severe, as defined by the Medical Dictionary for Regulatory Activities (version 11.0). Serious adverse events were designated as any event that resulted in death, disability, prolonged or new hospitalization, or were life threatening or required medical or surgical intervention. A 1.5-T MRI study was performed within 48 hours postoperatively and at 12 months. Adverse events were categorized as either related to the graft, graft procedure, DBS surgery, DBS therapy, or not related to either surgical or therapeutic component.

\section{Surgical Implantation}

The surgical components of stimulator placement and 
graft placement have been described in detail ${ }^{38}$ and are summarized in brief below. As is typical at our institution, DBS surgery takes place over two stages. ${ }^{39}$ Stage I involves the implantation of the pulse generator and extensions. Stage II is when the DBS electrodes are implanted. The trajectory of the stimulating electrode to the STN was planned based on visualized targeting. Preoperative susceptibility-weighted imaging (SWI) MR sequences were used for target localization, and T2-weighted and magnetization-prepared rapid acquisition gradient-echo sequences with contrast were used to establish trajectories that avoided the sulci, ventricles, and vasculature. Entry through the cortical gyri in the vicinity of the coronal suture allowed for lead trajectories that passed through the long axis of the STN. Plans were created using Brainlab software (iPlan Stereotaxy 3.0) for use with the CosmanRoberts-Wells frame (Integra). DBS surgeries were performed while the patient was awake with microelectrode recording and test stimulation. ${ }^{39}$

Once the stimulating electrodes were successfully placed, the graft was implanted unilaterally into the substantia nigra contralateral to the most affected side based on the UPDRS part III scores. The 5-mm-long target zone within the substantia nigra was located between $1 \mathrm{~mm}$ and $6 \mathrm{~mm}$ deep to the inferior border of the STN. The inferior border was identified intraoperatively by the electrophysiological activity that was recorded during stimulating electrode implantation. A guide cannula (1.8-mm-wide outer diameter; FHC, Inc.) was then placed within the graft target zone. The graft implantation trajectory was parallel to that of the stimulating electrode, at approximately $3 \mathrm{~mm}$ posterolateral, and did not require the creation of an additional burr hole.

The sural nerve that had been prepared and identified in stage $\mathrm{I}^{38,39}$ was then re-identified, and a 2 -cm-long segment of the nerve was harvested distal to the previous transection site. The nerve segment was rinsed in sterile saline, the epineurium was removed, and the fascicles were then cut to produce smaller, approximately 1-mmlong segments. Five to 6 segments were then loaded into the custom-made graft cannula, and the graft cannula was placed to the target through the same burr hole used for the stimulating electrode. After graft placement, the incisions were closed with subcutaneous sutures.

\section{Postoperative Evaluation}

After DBS and graft implantation, participant were admitted overnight. Each participant underwent postoperative MRI to confirm electrode placement and to evaluate the region of the graft target zone. Stimulator programming was initiated in the immediate postoperative period before discharge. Patients underwent clinical follow-up through routine postoperative visits, programming visits, and postoperative study visits. Medication changes were documented and reported as the levodopa equivalent dose (LED) ${ }^{29}$ For UPDRS testing, participants stopped taking medication at least 12 hours before undergoing UPDRS testing. After surgery, participants (if on medication) stopped medication 12 hours before UPDRS testing and turned off DBS 12 hours before UPDRS testing, except for the one participant (participant 2) who stopped tak- ing medication and turned off the DBS stimulator 3 hours before UPDRS testing.

\section{Neurocognitive Testing and Analysis}

The neurocognitive assessment battery was designed to meet the Movement Disorders Society's Task Force Guidelines for determining mild cognitive impairment and PD dementia., ${ }^{9,22}$ Level II criteria for comprehensive assessment of cognition in patients with PD include multiple measures of executive functioning, attention, working memory, language, memory, and visuospatial functions. Some subtests of these measures were used to assess both verbal and psychomotor speed. In addition, an estimate of premorbid function and a brief measure of general intelligence were included to aid clinical judgments of changes in individual cases and provide a comprehensive neurocognitive assessment. Affective and emotional symptoms were measured using a self-reported measure to complement clinical assessment during the patient interview. Postoperative evaluations were completed approximately 1 year after the DBS procedure (mean \pm SD $14.98 \pm 4.73$ months) using alternate forms of measures, where available, to minimize practice effects between assessments. The full assessment protocol for both the preoperative and postoperative evaluations involves a clinical interview with a neuropsychologist, with participation by both the participant and an informant, and battery administration by a clinically licensed, master's level psychometrician. All performances on the neurocognitive measures were quantified using appropriate normative sources, and all neuropsychological data were interpreted by a neuropsychologist. Analysis focused on individual changes between the preoperative and postoperative scores.

\section{Data Analysis}

Results were analyzed using the t-statistic (or Wilcoxon matched-pairs test for nonparametric values) for paired group means between the baseline and 12-month assessments (Prism 6.0, GraphPad Software Inc.). Mean values are presented with standard deviation. The minimal level of significance was $p \leq 0.05$ (2-tail criterion). Evaluation of the neurocognitive data were completed using IBM SPSS (version 23) with the majority of scores in standard form (mean 100, SD 15). Raw scores were compared as the affective measure. Due to the small sample size, the normality of the score distribution was examined, and the results suggested discordant normality for some scores (e.g., $\mathrm{p}=$ 0.03 for the presurgical riddles subtest of the Kaufman Brief Intelligence Test- 2 vs $p=0.10$ for the postsurgical results, Kolmogorov-Smirnov test). Given this finding, nonparametric analysis with the Wilcoxon signed-rank test was employed to examine the neurocognitive data. One participant was included in only some analyses because of differences in the presurgical battery construction.

\section{Results}

\section{Patient Summary}

Eight participants -6 males and 2 females - met the criteria for participation and successfully completed the study protocol. The mean participant age was $62.9 \pm 9.2$ 
years, and the mean disease duration was $9.8 \pm 3.9$ years. Because of family issues, 1 participant was not able to participant in the intermediate therapeutic evaluation visits (6 and 9 months) but was able to complete the 12-month evaluations. With respect to safety and adverse event reporting, the participant was evaluated by the local movement disorders neurologist and was in close communication with our study team throughout the study.

\section{Safety}

All adverse events were submitted to and reviewed by the DSMB at the University of Kentucky and are summarized in Table 1. The events listed include all events that presented from the first stage of the surgery through study closeout at 12 months postimplantation. One adverse event, superficial cellulitis surrounding the ankle incision, was graded as mild and categorized as serious and related to the graft procedure. The participant was treated with a 10-day course of antibiotics, and the cellulitis resolved. No other adverse events were related to graft harvesting or graft implantation. Two adverse events, urinary retention and headache, were mild, not serious, and related to the DBS surgery and resolved without further intervention. Three events were deemed to be mild, not serious, and not related and included falls and headache. One event, hypomania, was mild, not serious, and related to DBS stimulation and resolved promptly after changing the stimulation of the more proximal set of contacts of the stimulating electrodes. With respect to DBS surgery, there were no observed infections, hardware malfunctions, or skin erosions. With respect to feasibility, all participants underwent successful graft harvesting and implantation without complication or significant delay.

\section{MRI Data}

MR images (1.5 T) were obtained within 48 hours and at 12 months to evaluate the midbrain region for evidence of abnormalities, including swelling, edema, or contrast enhancement. The MR images from the 48-hour time point have been reported previously. ${ }^{38}$ There was no evidence of swelling or edema on the T2-weighted images and no evidence of enhancement on the T1-weighted contrast images within 48 hours or at 12 months. The MRI data also confirmed the appropriate placement of the DBS electrodes within the STN (Fig. 1). There was no evidence of complications, such as hemorrhage or stroke, along the trajectories of the electrodes.

\section{Clinical Measures}

A summary of the clinical data is included in Table 2, with individualized scores listed in Tables 3 and 4. Analysis of each of the 4 parts of UPDRS revealed that all parts showed a decrease (improvement) from baseline. Because UPDRS part III in the off state is the most appropriate score to evaluate any potential influence from the graft, we focused our subgroup analysis on the UPDRS part III scores. Collectively, the part III off scores at 12 months $(25.1 \pm 15.9$ points) decreased from baseline $(32.5 \pm 9.7$ points), but the difference was not statistically different (Table 2). We also individually scored each participant
TABLE 1. Cumulative 1-year adverse events

\begin{tabular}{|c|c|c|c|c|}
\hline $\begin{array}{l}\text { Case } \\
\text { No. }\end{array}$ & Event & $\begin{array}{c}\text { Significant } \\
\text { Adverse } \\
\text { Event }\end{array}$ & Status & Relatedness \\
\hline 1 & Urinary retention & No & Resolved & DBS surgery \\
\hline 2 & Hypomania & No & Resolved & Stimulation \\
\hline 3 & None & NA & NA & NA \\
\hline 4 & $\begin{array}{c}\text { Superficial cellulitis } \\
\text { of ankle incision }\end{array}$ & Yes & Resolved & Graft harvesting \\
\hline \multirow[t]{3}{*}{5} & Cough & No & Resolved & Not related \\
\hline & Headache & No & Resolved & DBS surgery \\
\hline & Falls & No & Resolved & Not related \\
\hline 6 & Fall \& head laceration & No & Resolved & Not related \\
\hline 7 & None & NA & NA & NA \\
\hline 8 & None & NA & NA & NA \\
\hline
\end{tabular}

$\mathrm{NA}=$ not applicable.

(Fig. 2) with respect to changes in baseline using values based on clinically important differences (CIDs). According to Shulman et al., ${ }^{34}$ minimal, moderate, and large CIDs are 2.5, 5.2, and 10.8 points from baseline, respectively.

We further analyzed the UPDRS part III data by performing subgroup evaluations of the collective data of all participants to investigate potential differences among symptom subsets, including tremor, rigidity, bradykinesia, and axial symptoms (speech, facial expression, gait, posture, postural instability, and body bradykinesia), and between lateralized scores relative to graft placement (ipsilateral vs contralateral). Subgroup analysis demonstrated larger improvements in tremor and rigidity and minor improvements in bradykinesia and axial symptoms (Fig. 3). The analysis of the lateralized scores showed a greater overall reduction in scores on the side contralateral to the graft than the ipsilateral side (Fig. 4).

PD medications were $87.9 \%$ lower after surgery than before $(854 \pm 556.0$ baseline vs $103 \pm 215.7$ LED at 12 months; $t(7)=4.22, \mathrm{p}=0.004$ ) (Fig. 5). The majority of this reduction was accounted for by 6 of 8 patients who were off all PD medications during the study period.

\section{Neurocognitive Data}

Analysis revealed that neurocognitive performance remained largely stable between the preoperative and postoperative time points, with some notable exceptions (Table 5). Some degree of improvement was postoperatively seen on the constructional praxis task (block design test of the Wechsler Adult Intelligence Scale-Fourth Edition; $T$ $=0, \mathrm{p}=0.04)$ with a large effect size $(r=-0.55)$. A subtle change was observed in the approach to the problem-solving task, on which responses became less nonsystematic and random at the postoperative time point (nonperseverative errors on the Wisconsin Card Sorting Test-64 Card Version; $T=3, \mathrm{p}=0.6$ ). This change approached statistical significance, and the effect size was large $(r=-0.50)$. The clinical significance of these changes is unknown because both may be influenced by repeated exposures to the stimuli and/or improvements in fine motor function. 

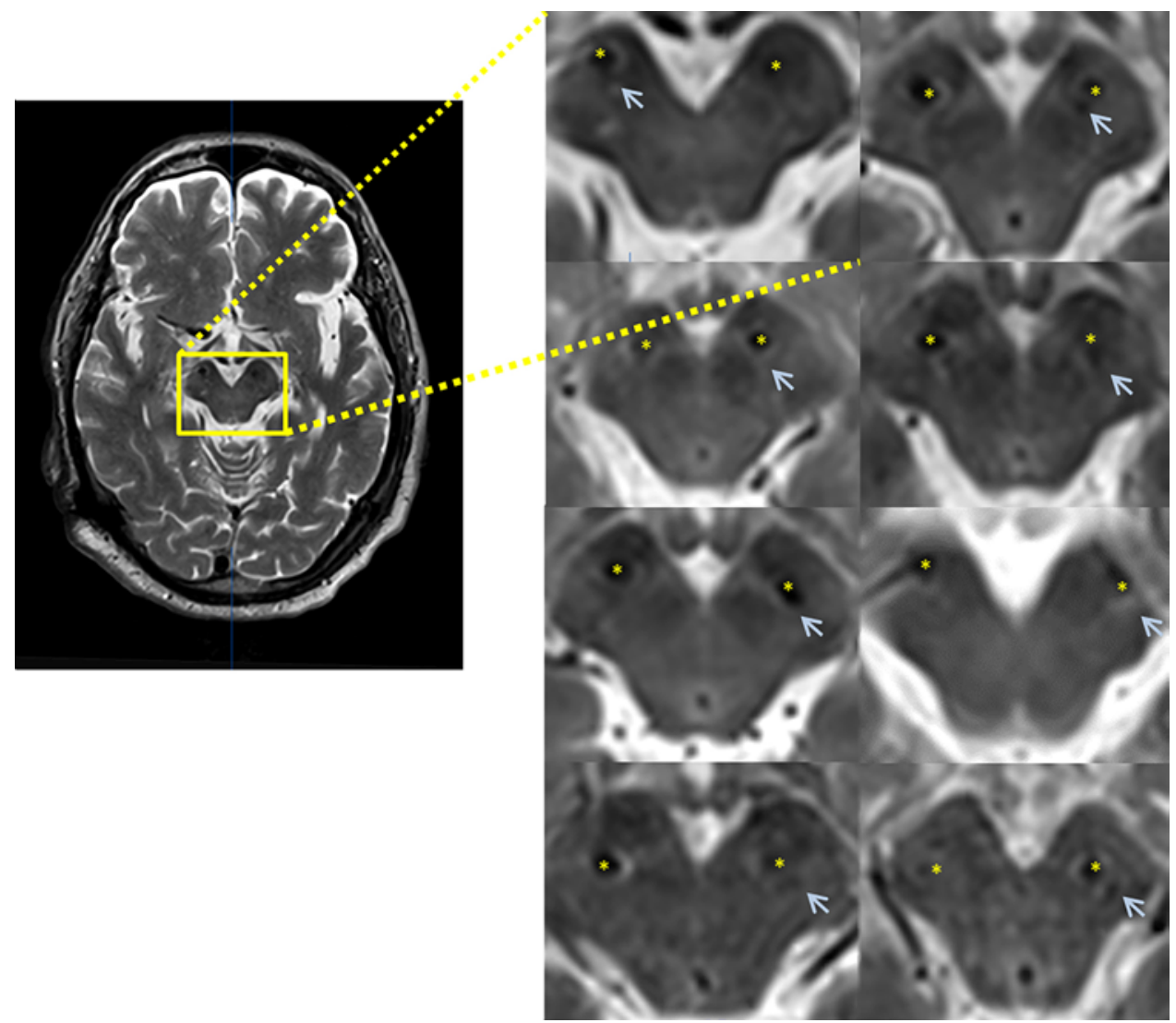

FIG. 1. Axial T2-weighted MR images obtained at 12 months in all participants, showing the area targeted for graft implantation (arrows). Asterisks indicate DBS electrode locations. Figure is available in color online only.

A significant decline was observed in phonemic fluency only (Controlled Oral Word Association Test; $T=0, \mathrm{p}=$ $0.02)$ with a large effect size $(r=-0.62)$. Levels of affective distress remained stable between the presurgical and postsurgical evaluations $(T=10, \mathrm{p}=0.92)$.

\section{Discussion}

As the first-ever reported attempt at combining a surgically delivered therapy with DBS, we designed this pilot study to test the safety and feasibility of this approach. Although we did not have a formal control group, we compared our results to similar patients in our database and to results in the literature where applicable. Specific risks for the procedure itself have been covered in detail in our previous publication, ${ }^{38}$ and as such we focus on the inclusive, longer term adverse events of the graft itself.

The adverse events collected throughout the study and monitored by our institutional DSMB showed only 1 serious adverse event that that could be attributed to the grafting procedure itself. This was superficial cellulitis at the graft harvest site that responded to oral antibiotics. The other adverse events reported were not related to the graft procedure or the graft and were similar to the adverse events reported by the DBS patients without a graft through 12 months (University of Kentucky DBS database; data not shown). Our total adverse events were also similar to those previously reported by other centers. ${ }^{10,30}$
TABLE 2. Participant scores

\begin{tabular}{|c|c|c|}
\hline \multirow[b]{2}{*}{ Variable } & \multicolumn{2}{|c|}{ Mean \pm SD } \\
\hline & Baseline & 12 Mos \\
\hline \multicolumn{3}{|l|}{ UPDRS } \\
\hline Part I* & $2.6 \pm 1.8$ & $2.1 \pm 1.7$ \\
\hline Part II† & $15.6 \pm 3.7$ & $14.5 \pm 5.8$ \\
\hline Part III & $32.5 \pm 9.7$ & $25.1 \pm 15.9$ \\
\hline Part III§ & $13.4 \pm 9.7$ & $10.9 \pm 9.7$ \\
\hline Part IVף & $5.5 \pm 1.9$ & $1.6 \pm 2.3$ \\
\hline \multicolumn{3}{|l|}{ Other scales } \\
\hline Modified Hoehn \& Yahr Scale** & $2.8 \pm 0.5$ & $2.8 \pm 0.7$ \\
\hline $\begin{array}{l}\text { Schwab \& England Activities of Daily } \\
\text { Living Scale†† }\end{array}$ & $75.0 \pm 9.3$ & $81.3 \pm 13.6$ \\
\hline Parkinson's Disease Questionnaire-8ł‡ & $9.4 \pm 4.4$ & $7.5 \pm 4.5$ \\
\hline
\end{tabular}

\footnotetext{
* Mentation, behavior, and mood (range 0-16).

$\dagger$ Activities of daily living while on therapy (range $0-52$ ).

‡ Motor examination while off therapy (range $0-108$ ).

$\S$ Motor examination while on therapy (range 0-108).

II Complications of therapy (range 0-23).

** Measured while off therapy (range $0-5$ ).

†† Range 0-100.

扯 Range $0-32$.
} 
TABLE 3. Individualized participant characteristics

\begin{tabular}{|c|c|c|c|c|c|c|c|c|c|c|c|c|}
\hline $\begin{array}{c}\text { Participant } \\
\text { No. }\end{array}$ & $\begin{array}{l}\text { Age } \\
\text { (yrs) }\end{array}$ & $\begin{array}{l}\text { Duration } \\
\text { w/ PD } \\
\text { (yrs) }\end{array}$ & $\begin{array}{l}\text { UPDRS } \\
\text { Part I } \\
\text { Score }\end{array}$ & $\begin{array}{l}\text { UPDRS } \\
\text { Part II } \\
\text { Score }\end{array}$ & $\begin{array}{l}\text { UPDRS } \\
\text { Part } \\
\text { III, On } \\
\text { Score }^{*}\end{array}$ & $\begin{array}{l}\text { UPDRS } \\
\text { Part } \\
\text { III, Off } \\
\text { Score† }\end{array}$ & $\begin{array}{l}\text { UPDRS } \\
\text { Part IV } \\
\text { Score }\end{array}$ & $\begin{array}{c}\text { UPDRS } \\
\text { Part IV } \\
\text { Score, } \\
\text { Section A } \ddagger\end{array}$ & $\begin{array}{l}\text { LED } \\
\text { (mg) }\end{array}$ & $\begin{array}{l}\text { Modified } \\
\text { Hoehn } \\
\text { \& Yahr } \\
\text { Scale }\end{array}$ & $\begin{array}{c}\text { Schwab \& } \\
\text { England } \\
\text { Activities of Daily } \\
\text { Living Scale }\end{array}$ & $\begin{array}{c}\text { Parkinson's } \\
\text { Disease } \\
\text { Questionnaire-8 }\end{array}$ \\
\hline \multicolumn{13}{|l|}{1} \\
\hline Baseline & 71 & 14 & 2 & 19 & 10 & 30 & 4 & 0 & 300 & 3 & 80 & 6 \\
\hline 12 mos & & & 5 & 18 & 13 & 32 & 0 & 0 & 0 & 4 & 60 & 8 \\
\hline \multicolumn{13}{|l|}{2} \\
\hline Baseline & 64 & 13 & 3 & 20 & 18 & 31 & 7 & 2 & 1800 & 3 & 70 & 11 \\
\hline 12 mos & & & 3 & 19 & 10 & $23 \S$ & 2 & 0 & 0 & 3 & 90 & 6 \\
\hline \multicolumn{13}{|l|}{3} \\
\hline Baseline & 55 & 6 & 6 & 9 & 30 & 48 & 8 & 3 & 900 & 3 & 70 & 17 \\
\hline 12 mos & & & 4 & 16 & 18 & 43 & 1 & 1 & 0 & 3 & 70 & 14 \\
\hline \multicolumn{13}{|l|}{4} \\
\hline Baseline & 48 & 12 & 4 & 16 & 5 & 34 & 3 & 1 & 375 & 3 & 80 & 11 \\
\hline 12 mos & & & 1 & 5 & 0 & 8 & 0 & 0 & 0 & 2 & 100 & 0 \\
\hline \multicolumn{13}{|l|}{5} \\
\hline Baseline & 56 & 5 & 2 & 12 & 11 & 28 & 3 & 0 & 500 & 2 & 90 & 4 \\
\hline 12 mos & & & 1 & 21 & 7 & 17 & 0 & 0 & 0 & 2 & 90 & 6 \\
\hline \multicolumn{13}{|l|}{6} \\
\hline Baseline & 75 & 14 & 3 & 18 & 6 & 28 & 6 & 3 & 1300 & 3 & 80 & 8 \\
\hline 12 mos & & & 2 & 11 & 6 & 17 & 1 & 1 & 225 & 3 & 80 & 5 \\
\hline \multicolumn{13}{|l|}{7} \\
\hline Baseline & 65 & 6 & 1 & 16 & 3 & 17 & 7 & 1 & 1280 & 2 & 60 & 13 \\
\hline 12 mos & & & 1 & 18 & 3 & 9 & 7 & 2 & 600 & 2 & 90 & 13 \\
\hline \multicolumn{13}{|l|}{8} \\
\hline Baseline & 69 & 8 & 0 & 15 & 24 & 44 & 6 & 1 & 380 & 3 & 70 & 5 \\
\hline 12 mos & & & 0 & 8 & 30 & 52 & 2 & 0 & 0 & 3 & 70 & 8 \\
\hline
\end{tabular}

* Baseline measurements were performed during the rising phase of the levodopa challenge. Measurements at 12 months were performed after the DBS had been on for at least 45 minutes and during the rising phase of the levodopa challenge (if applicable).

$\dagger$ Baseline measurements were performed after the PD medications had been stopped for $>12$ hours. Measurements at 12 months were performed after the DBS had been off for $>12$ hours and PD medications had been stopped for $>12$ hours (if applicable).

$\ddagger$ Measures dyskinesia on a scale of $0-13$.

$\S$ DBS had been off for only 3 hours at measurement.

MR images obtained at 12 months postoperatively showed no evidence of signal abnormality or contrast enhancement in the region of interest (ROI) based on preoperative targeting. These images were compared with the baseline and 24-hour postoperative MR images. Used in this way, MRI is able to provide information regarding potential adverse interactions of the graft with the surrounding parenchyma, such as inflammation, edema, or breakdown of the blood-brain barrier. MRI does have its limitations in relation to our protocol. Our ROI is very small, measuring only $1.5 \mathrm{~mm}$ in diameter, and the presence of a DBS electrode limits our scan potential to $1.5 \mathrm{~T}$. Additionally, the artifact from the electrode can be substantial, especially on SWI sequences. Functional MRI is not practical due to poor resolution with $1.5 \mathrm{~T}$ and the need for the patient to remain still for a prolonged period of time, which is especially difficult for many patients because the stimulator must be turned off during the scanning process. Although DaTscan ( ${ }^{123}$ I-ioflupane) analysis (GE Healthcare) does hold some promise for evaluating the potential effects on the dopaminergic terminal field in the striatum, the lack of dopamine transporters on the cell bodies in the substantia nigra precludes its use for directly evaluating the region. MRI remains a useful tool for evaluating tissue integrity within the ROI of the graft but lacks the utility for assessing graft survival or function.

\section{Clinical Data}

In this study, we report all components of the UPDRS examination. All components of our clinical evaluation, which are noted in Table 2, showed improvements, although none of the changes were statistically significant. Taken together, these results provide evidence that the grafting procedure in addition to DBS therapy does not negatively influence the therapeutic effect of DBS.

As noted previously, UPDRS part III is the most relevant clinical measure for evaluating potential influence from the graft; the scores from the other components of the examination are heavily influenced by the continual presence of DBS and medication therapy. Thus, the UP- 
TABLE 4. Individual lateralized scores while off medication and/or off stimulation

\begin{tabular}{|c|c|c|c|c|c|c|c|c|}
\hline \multirow{2}{*}{$\begin{array}{l}\text { Participant } \\
\text { No. }\end{array}$} & \multicolumn{2}{|c|}{ Total Lateralized Scores* } & \multicolumn{2}{|c|}{ Tremor† } & \multicolumn{2}{|c|}{ Rigidity } & \multicolumn{2}{|c|}{ Bradykinesiaß } \\
\hline & Ipsilateral & Contralateral & Ipsilateral & Contralateral & Ipsilateral & Contralateral & Ipsilateral & Contralateral \\
\hline \multicolumn{9}{|l|}{1} \\
\hline Baseline & 8 & 10 & 3 & 0 & 2 & 2 & 3 & 8 \\
\hline $12 \mathrm{mos}$ & 8 & 6 & $1 \rrbracket$ & $0^{* *}$ & $0 \pi$ & $0 \pi$ & $7 \dagger \dagger$ & $6 \pi$ \\
\hline \multicolumn{9}{|l|}{2} \\
\hline Baseline & 7 & 11 & 3 & 5 & 0 & 0 & 4 & 6 \\
\hline $12 \mathrm{mos}$ & 6 & 7 & $1 \rrbracket$ & $0 \rrbracket$ & $0^{* *}$ & $2 \dagger \dagger$ & $5 \dagger \dagger$ & $5 \pi$ \\
\hline \multicolumn{9}{|l|}{3} \\
\hline Baseline & 12 & 23 & 2 & 6 & 3 & 6 & 7 & 11 \\
\hline 12 mos & 12 & 19 & $2^{* *}$ & $4 \pi$ & $2 \pi$ & $3 \pi$ & $8 \dagger \dagger$ & $12 \dagger \dagger$ \\
\hline \multicolumn{9}{|l|}{4} \\
\hline Baseline & 10 & 12 & 4 & 7 & 0 & 2 & 6 & 3 \\
\hline 12 mos & 2 & 5 & $0 \rrbracket$ & $2 \pi$ & $0^{* *}$ & $1 \rrbracket$ & $2 \pi$ & $2 \pi$ \\
\hline \multicolumn{9}{|l|}{5} \\
\hline Baseline & 8 & 15 & 1 & 7 & 3 & 4 & 4 & 4 \\
\hline 12 mos & 5 & 7 & $1^{* *}$ & $2 \pi$ & $2 \pi$ & $2 \pi$ & $2 \uparrow$ & $3 \pi$ \\
\hline \multicolumn{9}{|l|}{6} \\
\hline Baseline & 7 & 11 & 0 & 2 & 1 & 1 & 6 & 8 \\
\hline 12 mos & 4 & 8 & $0^{* *}$ & $2^{* *}$ & $1^{* *}$ & $2 \nmid \dagger$ & $3 \pi$ & $4 \pi$ \\
\hline \multicolumn{9}{|l|}{7} \\
\hline Baseline & 5 & 7 & 0 & 1 & 1 & 1 & 4 & 5 \\
\hline 12 mos & 3 & 4 & $0^{* *}$ & $0 \rrbracket$ & $1^{* *}$ & $2 \dagger \dagger$ & $2 \rrbracket$ & $2 \pi$ \\
\hline \multicolumn{9}{|l|}{8} \\
\hline Baseline & 14 & 18 & 3 & 4 & 4 & 4 & 7 & 10 \\
\hline 12 mos & 20 & 14 & $4 \uparrow \dagger$ & $0 \rrbracket$ & $5+\dagger$ & $5 \dagger \dagger$ & $11 \dagger \dagger$ & $9 \rrbracket$ \\
\hline
\end{tabular}

* Range 0-36. Includes tremor at rest, action or postural tremor of hands, rigidity, finger taps, hand movements, rapid alternating movements of the hands, and leg agility.

† Range $0-12$. Includes tremor at rest and action or postural tremor of the hands.

$\ddagger$ Range $0-8$.

$\S$ Range 0-6. Includes finger taps, hand movements, rapid alternating movements of the hands, and leg agility.

II Improved scores from baseline.

** No change from baseline.

†† Worse score from baseline.

DRS part III off-therapy scores give a reasonable and practical way to measure a participant's baseline progression of motor symptoms.

Using each individual's preoperative scores as baseline, the composite scores of all participants showed an overall decline by 7.4 points. This compares to an expected annual increase of 3-5 points over time in patients with PD. ${ }^{14,19,40}$

In considering potential outcome measures for this and future studies that employ this paradigm, we chose to evaluate participants according to clinically important differences, as described previously.,34 The observed changes in the scores of the individual participants demonstrated that 6 of the participants scored lower (better) than baseline while 2 scored higher (worse). Using the UPDRS part III categorization for clinically important differences to assess the 6 participants who improved, 2 participants had a large CID, 3 had a moderate CID, and 1 had minimal CID.

Further analysis of the data subset showed that the biggest improvement in scores was in the domain of tremor. We do not think that this could be accounted for as a hold- over from DBS therapy because tremor is one of the first symptoms to return after DBS is turned off. ${ }^{37}$ In addition, we chose a more conservative DBS-off time interval of 12 hours for motor testing. Although 3 hours off DBS is adequate for off testing, ${ }^{37}$ we thought that an extended time would provide a more accurate assessment for scoring.

A comparison of unilateral versus contralateral scores showed improvements on both sides, but a larger improvement was noted on the side of the body contralateral to graft placement. Improvements unilateral to surgical therapies for PD have been reported for $\mathrm{DBS}^{5,41}$ and for the infusion of GDNF. ${ }^{35}$

We documented the change in PD medications in LDEs and report a large decrease in the total daily doses. The large variability is accounted for by the 2 participants who continued on their regimen with lower doses, whereas the other 6 participants remained off their medications for the 12 -month study period. We cannot determine whether the grafts had any impact on this finding. DBS of the STN allows for a substantial reduction in PD medication ${ }^{27} \mathrm{com}-$ 


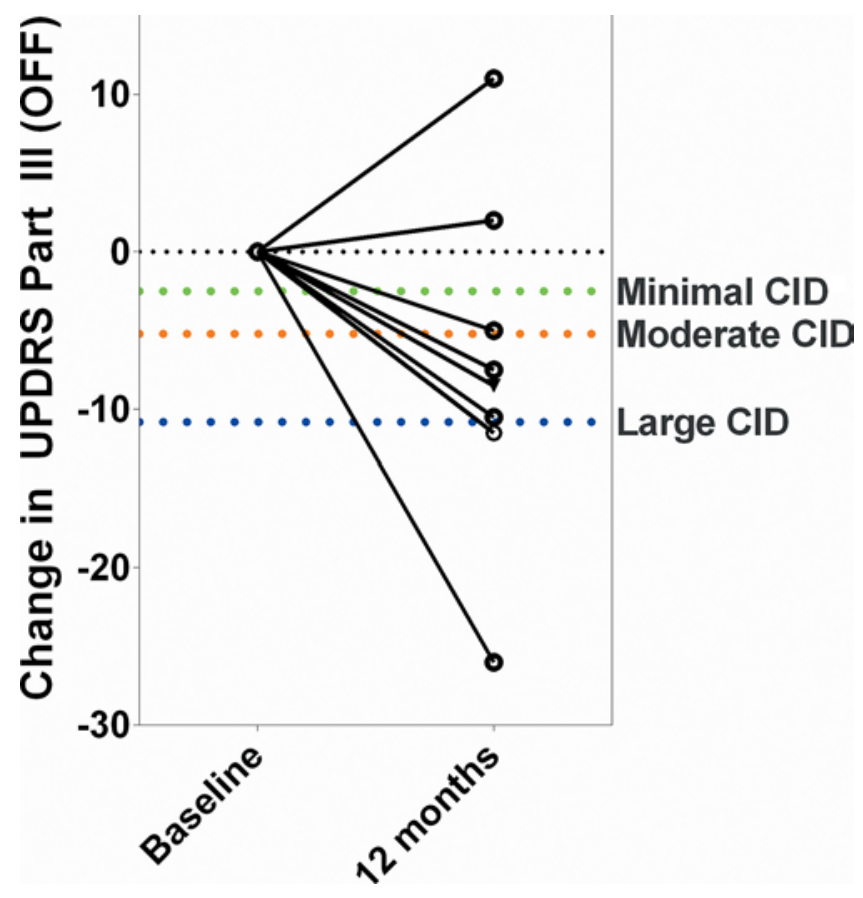

FIG. 2. Individual changes in motor scores (UPDRS part III) while off DBS and off medication therapy from baseline to 12 months after surgery. Minimal (2.5 points), moderate (5.2 points), and large (10.8 points) CIDs are indicated. Each symbol represents a different participant. Figure is available in color online only.

pared with DBS of the globus pallidus internus. ${ }^{42}$ We also note that with our protocol we implemented an intentionto-treat approach and therefore did not manipulate medications as indicated by the UPDRS part III results while DBS was on. As such, in future studies that evaluate potential disease-altering therapies in the setting of DBS therapy, we recognize that medication changes are important to monitor but are not a critical end point for measuring graft function.

\section{Neurocognitive Outcome}

Our analysis suggests that, despite some changes, postoperative neurocognitive functioning remained largely stable in this group and the levels of affective distress remained stable as well. Significant decline was limited to phonemic verbal fluency only, and this is commonly observed after DBS, in particular with STN stimulation. ${ }^{6}$ Potential improvements were observed in constructional praxis and abstract problem solving. However, these changes are of unknown clinical significance, as noted above, because these outcomes may have been affected by repeated exposure to these stimuli and improvements in fine motor functions. Furthermore, potential practice effects may have masked the underlying decline to some degree, although the true extent of that potential influence cannot be estimated accurately. However, because the time between evaluations was an average of more than 14 months, practice effects may have been less of a concern. In addition, the salient literature suggests that individuals with cognitive vulnerabilities such as mild cognitive impairment may not benefit from practice effects like control individuals without cognitive impairment.? Thus, practice effects may not have significantly influenced the current data, because some degree of mild cognitive impairment is prevalent in individuals with sufficient and severe PD who present for DBS. However, because this effect is difficult to quantify, the potential influence of repeated exposure to stimuli in the present data remains unknown. In any case, potential improvements in some skills, as expected, circumscribed the decline in specific neurocognitive functions, and stable neuropsychiatric status indicates encouraging safety and feasibility outcomes for this procedure.

\section{DBS Plus}

The positive safety and feasibility results presented in this project provide the first prospective, proof-of-concept evidence for the strategy of combining a cellular or biological therapy that is delivered in conjunction with DBS, al-

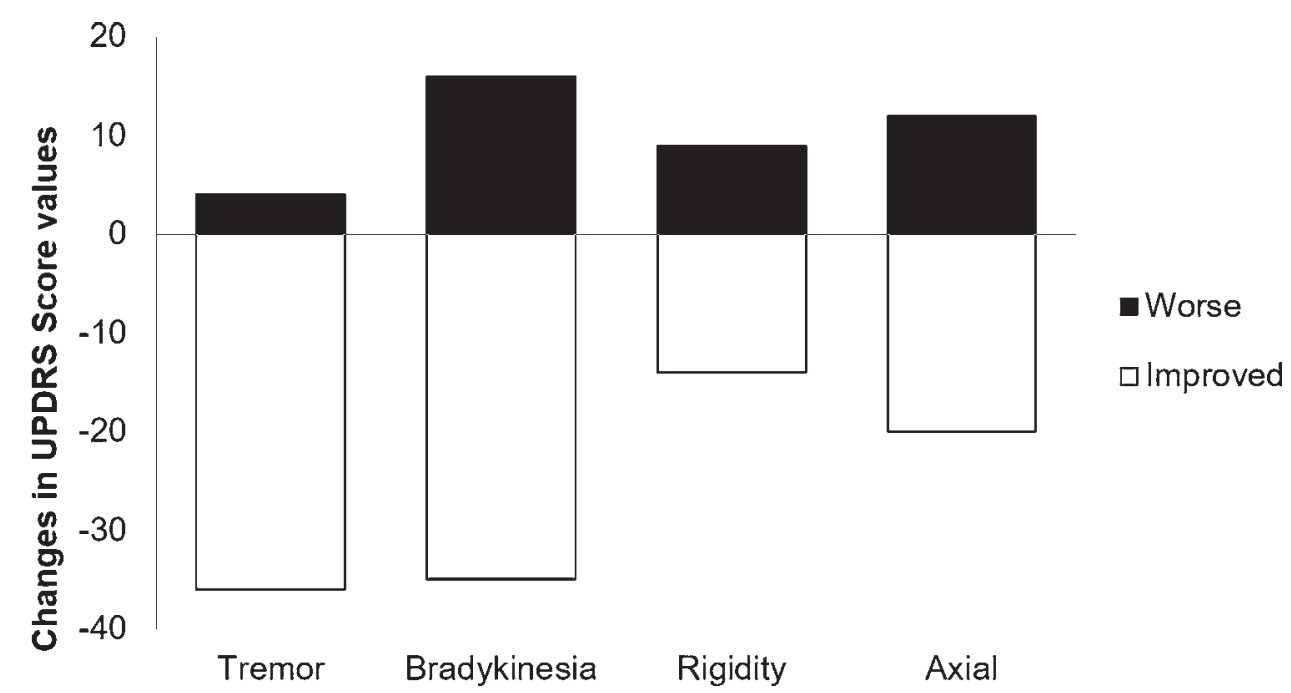

FIG. 3. For each of the domains of the UPDRS part III test, the total change in points for all participants was combined. The changes in the scores for all participants who performed worse in each domain were combined, and the changes in scores for all participants who showed improvements in each domain were combined. 


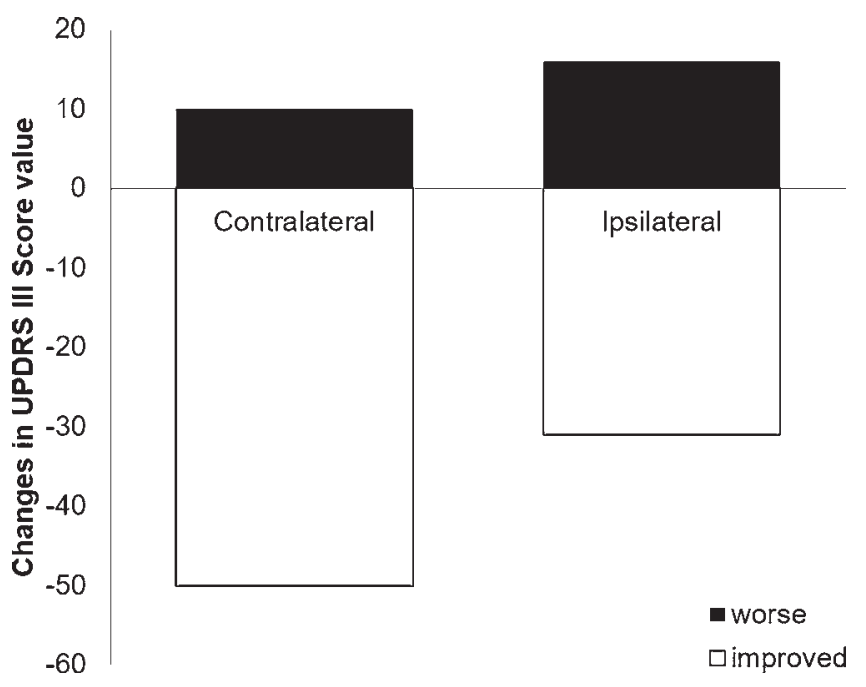

FIG. 4. Lateralized scores on the UPDRS part III test were used to identify differences in motor performance on the side contralateral or ipsilateral to graft placement. The changes in the scores of all participants who performed worse on the lateralized test were combined, and the changes in scores of all participants who showed improvements on the lateralized test were combined. Lateralized scores include tremor at rest, action or postural tremor of hands, rigidity, finger taps, hand movements, rapid alternating movements of the hand, and leg agility.

though several investigators have previously described the theoretical concept of combined therapy. ${ }^{8,32,33}$ Meanwhile, in several cases, some patients received DBS therapy after receiving cell therapy (fetal transplant after the development of runaway dyskinesias). ${ }^{13}$

We did not observe the development of off- or on-therapy dyskinesias in our patients through the course of our study or at any routine follow-up visits beyond the study timeline. Although it could be argued that the grafted tissue possibly caused the dyskinesias and the STN stimulation masked them, we did not observe any dyskinesias even during the 12 hours off stimulation at the testing time points. Thus, we report that tissue grafts can be implanted into the substantia nigra directly following the implantation of stimulating electrodes into the STN, and this adds minimal clinical risk for up to 1 year. Additionally, when performed in this manner, clinical evaluations for assessing graft function are straightforward and can be carried out easily and in accordance with current off-therapy protocols. Thus, we fully support the platform-which we have termed DBS Plus-for studying cellular or biological disease-modifying therapies in patients who are candidates for DBS therapy. The main advantage to the patients is that they are able to receive the full benefits of DBS therapy while participating in a clinical trial.

While it is possible that DBS therapy may influence graft function, as seen with the regional metabolic effects of DBS lead implantation, ${ }^{31}$ we think that testing this cellular therapeutic approach in patients receiving DBS is important. The overall clinical goal of any PD therapy is to improve patient outcomes. Given the complexity and clinical variability of $\mathrm{PD}$, it is unlikely that a new therapeutic approach will directly result in a frontline, monotherapeutic treatment. Rather, a more likely process is that a new therapeutic approach will at first become an adjunctive

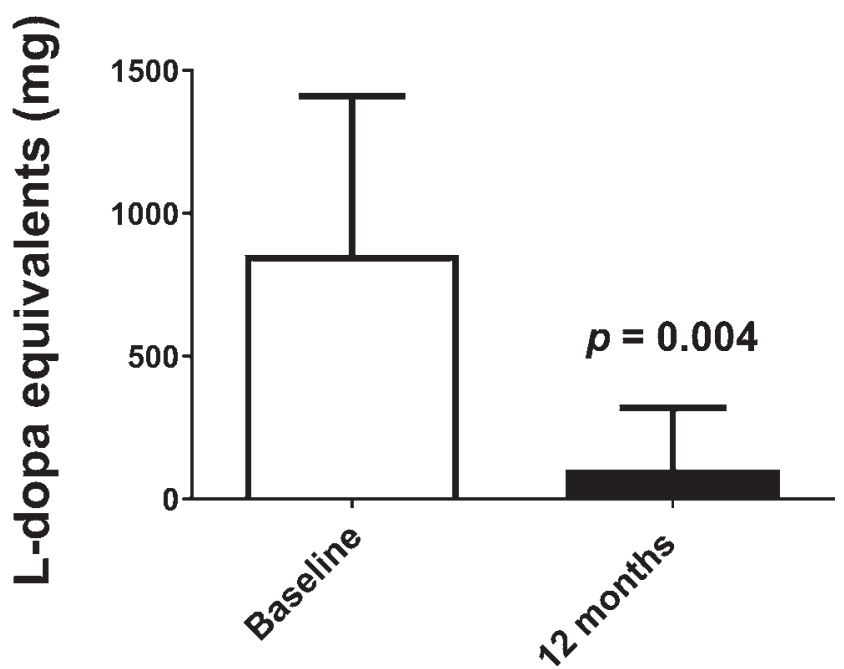

FIG. 5. Mean and SD (error bars) LEDs were significantly lower at 12 months postoperatively than before surgery. L-DOPA = levodopa.

therapy to current treatment strategies, much in the same way that DBS has become integrated into accepted adjunctive therapies for symptom control. If future studies show that an invasive, cellular, or biological approach could produce a disease-altering effect, then it could be appropriately tested in patients who are in earlier stages of illness.

Although we are proponents of the DBS Plus platform, there are limitations. For our study, the main limitation is our inability to track the survival and biological influence of the graft on the host environment. At this stage, we can only draw on the results of Kordower et al. ${ }^{20}$ who showed that sural nerve grafts to the nonhuman primate caudate and putamen contain surviving, nerve growth factor-rich Schwann cells at 3 months after grafting. As noted above, the ROI represented by the graft is very small and restricts the type of imaging, such as PET or SPECT scans, that could be beneficial. Because of the presence of the DBS system, MRI is limited to $1.5 \mathrm{~T}$, and with some sequences, such as SWI series, the artifact produced by the stimulating electrode may obscure the graft ROI. Our ongoing studies (www.clinicaltrials.gov, registration no. NCT02369003) have addressed some of these issues by including participants who are receiving DBS to the globus pallidus internus, thereby freeing the ROI of the graft from lead artifacts. We have also added DaTscan imaging to the protocol to investigate the possibility of the graft's influence on the nigral projections to the striatum. ${ }^{23}$ Another overall limitation of our current study is the inability to draw conclusions from the clinical efficacy data because of the small sample size. This is an expected limitation inherent in the design of this pilot study that focused on safety and feasibility. Other study design limitations include the lack of a control group and nonblinding, which does not take into account the possibility of the placebo effect on clinical measures. Furthermore, long-term follow-up will be required to fully assess the disease-altering capabilities, if any.

\section{Conclusions}

We present the results of our study in which we investigated the safety and feasibility of placing single, autolo- 
TABLE 5. Neurocognitive measures of various neurocognitive domains and skills

\begin{tabular}{|c|c|c|}
\hline Neurocognitive Measures* & Preop Mean \pm SD & Postop Mean \pm SD \\
\hline \multicolumn{3}{|l|}{ Premorbid functioning \& intelligence estimates } \\
\hline \multicolumn{3}{|l|}{ Premorbid status } \\
\hline Wide Range Achievement Test 4, word-reading subtest & $94.63 \pm 16.60$ & $95.05 \pm 18.23$ \\
\hline \multicolumn{3}{|l|}{ General intelligence } \\
\hline KBIT-2, intelligence quotient composite & $89.14 \pm 16.94$ & $88.43 \pm 14.91$ \\
\hline \multicolumn{3}{|l|}{ Verbal intelligence } \\
\hline KBIT-2, verbal & $88.57 \pm 16.32$ & $86.86 \pm 14.92$ \\
\hline \multicolumn{3}{|l|}{ Nonverbal \& perceptual intelligence } \\
\hline KBIT-2, nonverbal & $90.71 \pm 20.06$ & $90.71 \pm 16.76$ \\
\hline \multicolumn{3}{|l|}{ Verbal learning \& memory } \\
\hline \multicolumn{3}{|l|}{ List-based learning } \\
\hline HVLT-R, total recall & $81.37 \pm 15.01$ & $78.38 \pm 15.57$ \\
\hline \multicolumn{3}{|l|}{ List-based recall } \\
\hline HVLT-R, delayed recall & $85.63 \pm 18.70$ & $79.25 \pm 20.19$ \\
\hline \multicolumn{3}{|l|}{ List-based recognition memory } \\
\hline HVLT-R, recognition discrimination index & $97.12 \pm 10.37$ & $84.50 \pm 33.02$ \\
\hline \multicolumn{3}{|l|}{ Story-based learning } \\
\hline WMS-IV, logical memory I & $89.29 \pm 16.94$ & $85.71 \pm 14.27$ \\
\hline \multicolumn{3}{|l|}{ Story-based recall } \\
\hline WMS-IV, logical memory II & $81.14 \pm 16.29$ & $89.29 \pm 13.97$ \\
\hline \multicolumn{3}{|l|}{ Expressive \& receptive language } \\
\hline \multicolumn{3}{|l|}{ Confrontation naming } \\
\hline Boston Naming Test-2nd Edition & $87.43 \pm 25.08$ & $84.71 \pm 25.34$ \\
\hline \multicolumn{3}{|l|}{ Semantic fluency } \\
\hline Animal fluency & $92.38 \pm 17.24$ & $79.63 \pm 13.55$ \\
\hline \multicolumn{3}{|l|}{ Phonemic fluency } \\
\hline Controlled Oral Word Association Test & $91.29 \pm 15.81$ & $75.43 \pm 12.56 \dagger$ \\
\hline \multicolumn{3}{|l|}{ Expressive vocabulary } \\
\hline WAIS-IV, vocabulary subtest & $113.33 \pm 7.64$ & $102.50 \pm 8.66$ \\
\hline \multicolumn{3}{|l|}{ Auditory comprehension } \\
\hline Multilingual Aphasia Examination, token test & $98.00 \pm 17.94$ & $93.14 \pm 19.80$ \\
\hline \multicolumn{3}{|l|}{ Visuospatial skills } \\
\hline \multicolumn{3}{|l|}{ Spatial perception } \\
\hline Benton Judgment of Line Orientation & $89.63 \pm 17.25$ & $94.88 \pm 21.38$ \\
\hline \multicolumn{3}{|l|}{ Constructional praxis } \\
\hline WAIS-IV, block design subtest & $90.00 \pm 18.03$ & $95.00 \pm 18.03 \dagger$ \\
\hline \multicolumn{3}{|l|}{ Attention \& executive functions } \\
\hline \multicolumn{3}{|l|}{ Attention \& working memory } \\
\hline WAIS-IV, digit span subtest & $95.00 \pm 8.45$ & $93.75 \pm 12.17$ \\
\hline \multicolumn{3}{|l|}{ Mental flexibility \& divided attention } \\
\hline TMT-B & $82.57 \pm 33.22$ & $85.17 \pm 20.60$ \\
\hline Response inhibition & & \\
\hline Stroop, color-word & $91.29 \pm 12.54$ & $82.29 \pm 18.25$ \\
\hline Stroop, interference & $106.29 \pm 10.31$ & $100.29 \pm 16.61$ \\
\hline Abstract problem solving & & \\
\hline WCST-64, total error rate & $77.71 \pm 15.53$ & $85.43 \pm 17.82$ \\
\hline WCST-64, perseverative error rate & $86.43 \pm 14.07$ & $83.14 \pm 19.83$ \\
\hline WCST-64, nonperseverative error rate & $76.86 \pm 15.55$ & $95.86 \pm 13.23$ \\
\hline WCST-64, categories completed ${ }^{*}$ & $1.57 \pm 1.27$ & $2.14 \pm 1.77$ \\
\hline
\end{tabular}


» CONTINUED FROM PAGE 1559

TABLE 5. Neurocognitive measures of various neurocognitive domains and skills

\begin{tabular}{ccc}
\hline \multicolumn{1}{c}{ Neurocognitive Measures* } & Preop Mean \pm SD & Postop Mean \pm SD \\
\hline Information processing speed & & \\
\hline Verbal speed & $82.14 \pm 14.65$ & $79.71 \pm 15.01$ \\
\hline Stroop, word reading & $79.57 \pm 8.92$ & $75.57 \pm 15.24$ \\
\hline Stroop, color naming & & $85.29 \pm 15.73$ \\
\hline Psychomotor speed & $83.86 \pm 21.08$ & \\
\hline TMT-A & & \\
\hline Emotional functioning & & \\
\hline Psychiatric symptoms & $21.29 \pm 25.02$ & $26.50 \pm 27.36$ \\
\hline Psychiatric Diagnostic Screening Questionnaire, total score & & \\
\hline
\end{tabular}

HVLT-R = Hopkins Verbal Learning Test-Revised; KBIT = Kaufman Brief Intelligence Test; TMT = Trail Making Test; WAIS-IV = Wechsler Adult Intelligence Scale-

Fourth Edition; WCST-64 = Wisconsin Card Sorting Test-64 Card Version; WMS-IV = Wechsler Memory Scale-Fourth Edition.

* Values are provided as standard index scores with a mean \pm SD of $100 \pm 15$ unless otherwise noted with an asterisk.

$\dagger$ Significant at $p<0.05$.

gous, peripheral nerve grafts into the substantia nigra of patients with PD undergoing DBS therapy and report our clinical outcome measures. This study is unique in that it is the first to provide prospective data on using the DBS Plus platform that combines cellular therapy with DBS therapy, and this is the first study to evaluate the potential effects of peripheral nerve grafts on the progression of PD. We demonstrated that the procedure can be performed in a straightforward manner with minimal additional risk to patients for up to 1 year. In addition, we present preliminary evidence of the possible influence of the graft on motor measures (UPDRS part III) in the off state at 1 year. In the context of the inherent limitations of this pilot study design, we think that the overall positive findings presented here warrant further clinical investigation.

\section{Acknowledgments}

We thank Ann Hanley for her help and support with the study participants. Funding was provided by the National Center for Advancing Translational Sciences through National Institutes of Health grant UL1TR000117. The content is solely the responsibility of the authors and does not necessarily represent the official views of National Institutes of Health.

\section{References}

1. Arthur-Farraj PJ, Latouche M, Wilton DK, Quintes S, Chabrol E, Banerjee A, et al: c-Jun reprograms Schwann cells of injured nerves to generate a repair cell essential for regeneration. Neuron 75:633-647, 2012

2. Barker RA, Barrett J, Mason SL, Björklund A: Fetal dopaminergic transplantation trials and the future of neural grafting in Parkinson's disease. Lancet Neurol 12:84-91, 2013

3. Bartus RT, Baumann TL, Brown L, Kruegel BR, Ostrove JM, Herzog CD: Advancing neurotrophic factors as treatments for age-related neurodegenerative diseases: developing and demonstrating "clinical proof-of-concept" for AAV-neurturin (CERE-120) in Parkinson's disease. Neurobiol Aging 34:35-61, 2013

4. Caplan LR: How well does "evidence-based" medicine help neurologists care for individual patients? Rev Neurol Dis 4:75-84, 2007

5. Chung SJ, Jeon SR, Kim SR, Sung YH, Lee MC: Bilateral ef- fects of unilateral subthalamic nucleus deep brain stimulation in advanced Parkinson's disease. Eur Neurol 56:127-132, 2006

6. Combs HL, Folley BS, Berry DTR, Segerstrom SC, Han DY, Anderson-Mooney AJ, et al: Cognition and depression following deep brain stimulation of the subthalamic nucleus and globus pallidus pars internus in Parkinson's disease: a metaanalysis. Neuropsychol Rev 25:439-454, 2015

7. Cooper DB, Lacritz LH, Weiner MF, Rosenberg RN, Cullum CM: Category fluency in mild cognitive impairment: reduced effect of practice in test-retest conditions. Alzheimer Dis Assoc Disord 18:120-122, 2004

8. During MJ, Kaplitt MG, Stern MB, Eidelberg D: Subthalamic GAD gene transfer in Parkinson disease patients who are candidates for deep brain stimulation. Hum Gene Ther 12:1589-1591, 2001

9. Emre M, Aarsland D, Brown R, Burn DJ, Duyckaerts C, Mizuno Y, et al: Clinical diagnostic criteria for dementia associated with Parkinson's disease. Mov Disord 22:16891707, 1837, 2007

10. Fenoy AJ, Simpson RK Jr: Risks of common complications in deep brain stimulation surgery: management and avoidance. J Neurosurg 120:132-139, 2014

11. Funakoshi H, Frisén J, Barbany G, Timmusk T, Zachrisson O, Verge VM, et al: Differential expression of mRNAs for neurotrophins and their receptors after axotomy of the sciatic nerve. J Cell Biol 123:455-465, 1993

12. Gill SS, Patel NK, Hotton GR, O'Sullivan K, McCarter R, Bunnage M, et al: Direct brain infusion of glial cell linederived neurotrophic factor in Parkinson disease. Nat Med 9:589-595, 2003

13. Graff-Radford J, Foote KD, Rodriguez RL, Fernandez HH, Hauser RA, Sudhyadhom A, et al: Deep brain stimulation of the internal segment of the globus pallidus in delayed runaway dyskinesia. Arch Neurol 63:1181-1184, 2006

14. Guimaraes P, Kieburtz K, Goetz CG, Elm JJ, Palesch YY, Huang P, et al: Non-linearity of Parkinson's disease progression: implications for sample size calculations in clinical trials. Clin Trials 2:509-518, 2005

15. Henderson CE, Phillips HS, Pollock RA, Davies AM, Lemeulle C, Armanini M, et al: GDNF: a potent survival factor for motoneurons present in peripheral nerve and muscle. Science 266:1062-1064, 1994

16. Jessen KR, Mirsky R: The repair Schwann cell and its function in regenerating nerves. J Physiol 594:3521-3531, 2016

17. Kalia LV, Lang AE: Parkinson's disease. Lancet 386:896912, 2015 
18. Kidd GJ, Ohno N, Trapp BD: Biology of Schwann cells, in Gérard S, Christian K (eds): Handbook of Clinical Neurology. Amsterdam: Elsevier, 2013, Vol 115, pp 55-79

19. Kieburtz K: Designing neuroprotection trials in Parkinson's disease. Ann Neurol 53 (Suppl 3):S100-S109, 2003

20. Kordower JH, Fiandaca MS, Notter MF, Hansen JT, Gash DM: NGF-like trophic support from peripheral nerve for grafted rhesus adrenal chromaffin cells. J Neurosurg 73:418-428, 1990

21. Lang AE, Gill S, Patel NK, Lozano A, Nutt JG, Penn R, et al: Randomized controlled trial of intraputamenal glial cell linederived neurotrophic factor infusion in Parkinson disease. Ann Neurol 59:459-466, 2006

22. Litvan I, Goldman JG, Tröster AI, Schmand BA, Weintraub D, Petersen RC, et al: Diagnostic criteria for mild cognitive impairment in Parkinson's disease: Movement Disorder Society Task Force guidelines. Mov Disord 27:349-356, 2012

23. Marek K, Innis R, van Dyck C, Fussell B, Early M, Eberly S, et al: ${ }^{123}$ I] $\beta$-CIT SPECT imaging assessment of the rate of Parkinson's disease progression. Neurology 57:2089-2094, 2001

24. Marks WJ Jr, Bartus RT, Siffert J, Davis CS, Lozano A, Boulis N, et al: Gene delivery of AAV2-neurturin for Parkinson's disease: a double-blind, randomised, controlled trial. Lancet Neurol 9:1164-1172, 2010

25. Marks WJ Jr, Ostrem JL, Verhagen L, Starr PA, Larson PS, Bakay RA, et al: Safety and tolerability of intraputaminal delivery of CERE-120 (adeno-associated virus serotype 2-neurturin) to patients with idiopathic Parkinson's disease: an open-label, phase I trial. Lancet Neurol 7:400-408, 2008

26. Meyer M, Matsuoka I, Wetmore C, Olson L, Thoenen H: Enhanced synthesis of brain-derived neurotrophic factor in the lesioned peripheral nerve: different mechanisms are responsible for the regulation of BDNF and NGF mRNA. J Cell Biol 119:45-54, 1992

27. Molinuevo JL, Valldeoriola F, Tolosa E, Rumia J, Valls-Sole J, Roldan H, et al: Levodopa withdrawal after bilateral subthalamic nucleus stimulation in advanced Parkinson disease. Arch Neurol 57:983-988, 2000

28. Olanow CW, Goetz CG, Kordower JH, Stoessl AJ, Sossi V, Brin MF, et al: A double-blind controlled trial of bilateral fetal nigral transplantation in Parkinson's disease. Ann Neurol 54:403-414, 2003

29. Pahwa R, Wilkinson SB, Overman J, Lyons KE: Bilateral subthalamic stimulation in patients with Parkinson disease: long-term follow up. J Neurosurg 99:71-77, 2003

30. Patel DM, Walker HC, Brooks R, Omar N, Ditty B, Guthrie BL: Adverse events associated with deep brain stimulation for movement disorders: analysis of 510 consecutive cases. Neurosurgery 11 (Suppl 2):190-199, 2015

31. Pourfar M, Tang C, Lin T, Dhawan V, Kaplitt MG, Eidelberg D: Assessing the microlesion effect of subthalamic deep brain stimulation surgery with FDG PET. J Neurosurg 110:1278-1282, 2009

32. Rowland NC, Kalia SK, Kalia LV, Larson PS, Lim DA, Bankiewicz KS: Merging DBS with viral vector or stem cell implantation: "hybrid" stereotactic surgery as an evolution in the surgical treatment of Parkinson's disease. Mol Ther Methods Clin Dev 3:15051, 2016

33. Rowland NC, Starr PA, Larson PS, Ostrem JL, Marks WJ Jr, Lim DA: Combining cell transplants or gene therapy with deep brain stimulation for Parkinson's disease. Mov Disord 30:190-195, 2015

34. Shulman LM, Gruber-Baldini AL, Anderson KE, Fishman PS, Reich SG, Weiner WJ: The clinically important difference on the unified Parkinson's disease rating scale. Arch Neurol 67:64-70, 2010
35. Slevin JT, Gerhardt GA, Smith CD, Gash DM, Kryscio $\mathrm{R}$, Young B: Improvement of bilateral motor functions in patients with Parkinson disease through the unilateral intraputaminal infusion of glial cell line-derived neurotrophic factor. J Neurosurg 102:216-222, 2005

36. Taylor JS, Bampton ET: Factors secreted by Schwann cells stimulate the regeneration of neonatal retinal ganglion cells. J Anat 204:25-31, 2004

37. Temperli P, Ghika J, Villemure JG, Burkhard PR, Bogousslavsky J, Vingerhoets FJ: How do parkinsonian signs return after discontinuation of subthalamic DBS? Neurology 60:78-81, 2003

38. van Horne CG, Quintero JE, Gurwell JA, Wagner RP, Slevin JT, Gerhardt GA: Implantation of autologous peripheral nerve grafts into the substantia nigra of subjects with idiopathic Parkinson's disease treated with bilateral STN DBS: a report of safety and feasibility. J Neurosurg 126:1140-1147, 2017

39. van Horne CG, Vaughan SW, Massari C, Bennett M, Asfahani WS, Quintero JE, et al: Streamlining deep brain stimulation surgery by reversing the staging order. J Neurosurg 122:1042-1047, 2015

40. Venuto CS, Potter NB, Dorsey ER, Kieburtz K: A review of disease progression models of Parkinson's disease and applications in clinical trials. Mov Disord 31:947-956, 2016

41. Walker HC, Watts RL, Guthrie S, Wang D, Guthrie BL: Bilateral effects of unilateral subthalamic deep brain stimulation on Parkinson's disease at 1 year. Neurosurgery 65:302310,2009

42. Williams NR, Foote KD, Okun MS: STN vs. GPi deep brain stimulation: translating the rematch into clinical practice. Mov Disord Clin Pract (Hoboken) 1:24-35, 2014

\section{Disclosures}

This work was supported by gifts from the Brain Restoration Center, Tom Dupree for Parkinson's Disease Research, and Pro's Players Fore Parkinson's.

Dr. Gerhardt: previously received research support from Medtronic. Dr. Slevin: received educational support grants from Medtronic. Dr. van Horne: received educational support grants from Medtronic.

\section{Author Contributions}

Conception and design: van Horne, Quintero, Slevin, Gerhardt, Anderson-Mooney. Acquisition of data: van Horne, Quintero, Slevin, Anderson-Mooney, Gurwell, Wagner. Analysis and interpretation of data: van Horne, Quintero, Slevin, Anderson-Mooney, Gerhardt. Drafting the article: van Horne, Quintero, AndersonMooney, Lamm. Critically revising the article: van Horne, Quintero, Anderson-Mooney, Lamm, Gerhardt. Reviewed submitted version of manuscript: all authors. Approved the final version of the manuscript on behalf of all authors: van Horne. Statistical analysis: Quintero, Anderson-Mooney. Administrative/technical/ material support: Wagner. Study supervision: van Horne.

\section{Supplemental Information}

\section{Previous Presentations}

Portions of this work were presented in abstract and poster forms at the 20th International Congress of Parkinson's Disease and Movement Disorders, Berlin, Germany, June 19-23, 2016.

\section{Correspondence}

Craig G. van Horne: University of Kentucky, Lexington, KY. craigvanhorne@uky.edu. 